\title{
Mathematics for natural hierarchy
}

A new way of tackling the statistical problems of spin glasses relies on almost forgotten mathematics, but may have a bearing on problems in biology still unsolved.

How does it so often arise that the solution of newly defined problems hangs on the use of mathematical techniques independently developed, often decades earlier, for quite different purposes or (mathematicians being what they are) for no purpose at all? That question is the starting point for a delightful article in $R e$ views of Modern Physics for July this year in which the concept of "ultrametricity" is offered as an illustration of the principle that essential tools will usually be found lying discarded in the attics of the mathematicians. It goes without saying that the real world is not always as bountiful.

Part of the charm of this winsome piece is that its authors, R. Rammal (from the CNRS low-temperature institute at Grenoble), G. Toulouse (from the École Supérieure de Physique et Chemie Industrielles in Paris) and M.A. Virasoro (of the physics department of the University of Rome), confess that they themselves have only recently stumbled on the notion of ultrametricity, in an article on spin glasses in another physics journal. Like new converts in most fields, they have become enthusiastic proselytizers for the cause (Rev. Mod. Phys. 58, 765; 1986).

Historically, so far as Rammal et al. have been able to tell, ultrametricity goes back to 1897 , when the German mathematician Kurt Hensel published a recondite piece of algebraic number theory showing, among other things, that an arbitrary integer can always be represented as a sum of ascending powers of some other arbitrary integer, with each term in the series multiplied by a coefficient which may be zero but which is, in any case, less than the base for the ascending power series.

Nowadays, the most familiar illustration of the truth of Hensel's principle is the practice well known to computer buffs of representing decimal integers in binary form, which is merely a way of representing an arbitrary integer as a sum of powers of 2 in which sum the coefficients may be either 0 or 1 . The more general statement is that the base of daily arithmetic may be changed from 10 to any other arbitrary base. A further generalization is that rational numbers (with finite, or nonrecurring, decimal representation) may always be represented by the sum of powers of some arbitrarily chosen base in which some negative as well as positive powers are included. Rammal et al. say that Hensel's work was done in complete isolation, but its practical importance now cannot be gainsaid.

The other mathematical innovation from which ultrametricity has sprung is the formal definition of metric spaces at the turn of the century. For a 'space', or a set of points, to be metric, there must be some function called distance of any pair of points which satisfies the condition that the value of the function is zero when the two points are the same, and that the value is unaltered when any pair of points is interchanged.

Euclidean verities come to mind, together with the euclidean principle that the sum of the distances between one point and a second and between the second and third is greater than the direct distance between the first and the third. This is just another way of saying that, in the euclidean world, the sum of the lengths of two sides of a triangle (say $A B$ plus $B C$ ) is greater than the length of the third side $(A C)$. Ultrametricity requires a more stringent principle, that $A C$ should be less than whichever is the greater of $A B$ and $B C$.

Such a metric world is, of course, an odd world. Rammal et al. point to some formal mathematics in the 1940 s showing that, with definitions of metric distance satisfying these conditions, any point inside a sphere (defined as the region distant less than some fixed amount from a fixed point) will be itself at the centre of the sphere, and that the only possible triangles will be either equilateral or isosceles (in which case the base must be shorter than the two equal sides). Hensel's work is relevant because it provided one of the first illustrations of how such a metric might be defined.

What relevance can such an odd way of defining distance have to the problems of the real world? Rammal et al. start with a phylogenetic tree such as any cladist might construct. The tree implies a hierarchy which, once drawn, implies metric distances between the members at the lowest level, most easily thought of as the length of time since any pair of them diverged from a common ancestor. Then, in the comparison of the distances between three arbitrary extant species, either one pair has more recently evolved from a common ancestor than that evolved from some more distant common ancestor (in which case the triangle is isosceles) or no two of the three species have a common ancestor earlier than the putative common ancestor (in which case the triangle of the distances between them is equilateral in the ultrametric sense).

From this point, it is an easy step to the application of the ultrametric concept to all kinds of intriguing problems in data processing, especially to the use of treesorting algorithms. But Rammal and his colleagues are proudest of their novel application of ultrametricity to the problem of spin glasses, the structures (real enough) represented by three-dimensional lattices whose vertices are occupied by spins (think of them as little magnets) and whose energy is the sum of the magnetic interactions between all possible pairs of vertices in the lattice. The problem is that of an Ising lattice in which the simplifying assumption that only nearest neighbours interact is abandoned.

Experimentally, the spin glasses are peculiar materials, especially at low temperatures. Phase transitions occur to states in which the solid material appears to consist of small domains of magnetically ordered spins, but the degree of hysteresis associated with changing external circumstances (such as temperature) is unusual; the materials seem to have a capacity for long-term memory of their previous state. In the decade since the problem of the spin glasses was recognized as such, much has been done by analytical and numerical approximations to show that the low-temperature condition of such a material may be one of a great number of essentially similar configurations that are distinguishable from each other largely by their symmetry. What Rammal et al. are able to show is that these configurations are related to each other hierarchically, by means of an ultrametric metric.

That will be an interesting landmark, but will it be an important one? Much depends on whether the authors' speculations turn out to be realistic. They claim, for example, that their line of argument may have a bearing on the travelling salesman problem (how to travel to $N$ cities most economically), the founding conundrum of linear programming. Naturally, they say there is a place for neurobiology in their scheme of things. And since the folding of protein molecules into biologically active materials is a hierarchical process, why not subsume that as well? If they are right, a great many people will be learning a great deal of mathematics they did not previously know existed.

John Maddox 